\title{
The impact of COVID-19 pandemic on the rate of newly diagnosed gynecological and breast cancers: a tertiary center perspective
}

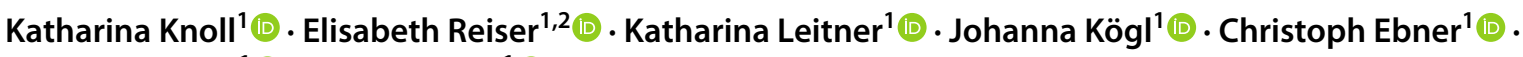 \\ Christian Marth ${ }^{1}$ (1) $\cdot$ Irina Tsibulak ${ }^{1}$
}

Received: 12 May 2021 / Accepted: 14 September 2021 / Published online: 24 September 2021

(c) The Author(s) 2021

\begin{abstract}
Purpose The aim of the present study was to assess the impact of postponed screening examinations and lockdown measures on gynecological and breast cancer diagnoses throughout the year 2020 in a gynecological oncological center in Austria.

Methods Data of 889 patients with either newly diagnosed gynecological or breast cancer between January 2019 and December 2020 were collected. Clinical parameters including symptoms, performance status, comorbidities and referral status were compared in patients, who were newly diagnosed with cancer in the period of the first lockdown from March 2020 to April 2020 and the second lockdown from November 2020 to December 2020 and compared to the same period in 2019. Results Our results showed a strong decline in newly diagnosed cancers during the lockdown periods: $-45 \%$ in gynecological cancer and $-52 \%$ in breast cancer compared to the same period in 2019. Compared to the analogue period of 2019, breast cancer patients reported significantly more tumor-associated symptoms (55\% vs. $31 \%, p=0.013)$ during and in between ( $48 \%$ vs. $32 \%, p=0.022$ ) the lockdowns. During the lockdown, periods in the group of breast cancer patients' tumor stage varied significantly compared to 2019 (T2-T4; $p=0.047$ ).

Conclusion Both lockdowns led to a strong decrease in newly diagnosed gynecological and breast cancers. Treatment delays in potentially curable disease could lead to inferior clinical outcomes, with the risk of missing the optimal treatment window. As the COVID-19 pandemic will be a challenge for some time to come, new strategies in patient care are needed to optimize cancer screening and management during the pandemic.
\end{abstract}

Keywords Gynecology $\cdot$ Carcinoma $\cdot$ Neoplasms $\cdot$ Breast cancer $\cdot$ COVID-19

\section{Introduction}

The ongoing coronavirus disease 2019 (COVID-19) pandemic remains a challenge for most countries around the world, affecting not only their healthcare system, but also economic development, socio-political attitude, educational and cultural sectors. As of 26 January 2021, more than 100 million infections were reported worldwide with a

Katharina Knoll and Elisabeth Reiser Contributed equally.

Irina Tsibulak

irina.tsibulak@i-med.ac.at

1 Department of Obstetrics and Gynecology, Medical University Innsbruck, Anichstrasse 35, Innsbruck, Austria

2 Department of Gynecological Endocrinology and Reproductive Medicine, Medical University Innsbruck, Innsbruck, Austria death toll of more than 2 million [1]. In Austria, more than 402000 cases and 7416 deaths were confirmed by 26 January 2020 , leading to a death rate of $1.8 \%$ [2].

The first two patients in Innsbruck, Austria, were diagnosed with (COVID-19) on 25 February 2020. Within few weeks, the number of cases increased throughout the country, leading the government to the implementation of a nationwide hard lockdown on 16 March 2020, closing all non-essential businesses and switching to distance learning in schools and universities. During the first wave of the COVID-19 pandemic, Austria's governmental measures prevented an overload of the healthcare system, as the maximum intensive care bed occupancy only reached $26 \%$ [2]. By the middle of April, the stay-at-home orders started to be loosened. The first lockdown ended on April $30^{\text {st }}$ enabling most of public life by the beginning of May. However, regained freedom of travel and growing get-together trend led to a new escalation of newly diagnosed COVID-19 
cases, so that a nationwide curfew was announced on 21 September, followed by the second lockdown enforced from 3 November.

In Austria, general practitioners and specialists play a central role in preventive and primary healthcare and they refer patients to hospitals, if necessary. Gynecological screening examinations and routine checkups in Austria include annual PAP smear testing and biennial mammography. During the first lockdown, these screening examinations and routine checkups were disrupted to prevent the spread of the virus and to increase the hospitals' bed capacity across the country [3]. These restrictions directly affected the tertiary centre as less patients were referred to the department with suspicious results and this led to a strong decline in newly diagnosed gynaecological and breast cancers in Austria, as already reported (-24\% in March 2020 vs. March 2019, $-49 \%$ in April 2020 vs. April 2019, $-49 \%$ in May 2020 vs. May 2019) [3]. Such a dramatic decrease in cancer diagnoses reflects the failures of pandemic management and, therefore, underlines the pivotal role of the gynecological screening examinations and routine checkups. After the first lockdown, significant efforts were made to relaunch health prevention and to promote the importance of screening programs during the pandemic. Furthermore, the safety of patients in hospitals was improved, as mandatory COVID-19 testing was implemented for every admitted patient as well as weekly screening of healthcare workers. During the second lockdown, screening examinations and routine checkups (PAP, biennial mammography) were maintained and access to the hospital, general practitioners and specialists was controlled by COVID-19 rapid testing and temperature measurements.

The aim of this study was to assess the impact of the COVID-19 pandemic and its management throughout the year 2020 on the rate of newly diagnosed gynecological and breast cancers from the perspective of a tertiary referral center in Innsbruck (federal state of Tyrol, Austria).

\section{Materials and methods}

\section{Patients}

All patients diagnosed with gynecological or breast cancer at the Department of Obstetrics and Gynecology, Medical University of Innsbruck in the period from January 2019 to December 2020, were included in the present study. The primary endpoint was the rate of newly diagnosed gynecological and breast cancer during the lockdown periods in 2020 and during the same time period in 2019. Gynecological cancer was defined as one of the following diagnoses: ovarian or tubal carcinoma, cervical carcinoma, endometrial carcinoma, uterine sarcoma, vulvar carcinoma, vaginal carcinoma, and germ cell tumors. Ductal carcinoma in situ (DCIS) of the breast was included in the breast cancer cohort. Clinical parameters such as tumor-specific symptoms, performance status, leading comorbidities, tumor type, tumor stage, referral status and COVID-19 infection status were the secondary endpoints. Referral status was defined as followed: referral by a specialist with or without symptoms, or self-determined presentation at emergency or gynecological department with or without tumor-specific symptoms. Leading comorbidities were classified as: cardiovascular, malignant, respiratory, infectious, rheumatic, endocrine and metabolic, psychiatric disease and others. Frontline therapy included surgery, neoadjuvant chemotherapy, neoadjuvant endocrine therapy, radiotherapy, chemoradiation and palliative therapy. Tumor stage was defined according to the Fédération Internationale de Gynécologie et d'Obstétrique (FIGO) staging system for gynecological tumors and according to TNM staging system for breast cancer.

The following symptoms have been regarded as tumorassociated: abdominal swelling/pain, abnormal vaginal bleeding (postmenopausal bleeding, menorrhagia, metrorrhagia, spotting) and palpable mass of the breast.

To assess the impact of the COVID-19 lockdown measures on the rate of newly diagnosed gynecological and breast tumors.

Subgroup analyses were performed in patients, who were newly diagnosed with cancer during the lockdown periods (the first lockdown from 16 March 2020 to 30 April 2020 and the second lockdown from 3 November 2020 to 31 December 2020) and compared to the same period in 2019 (16 March 2019 to 30 April 2019 and 3 November 2019 to 31 December 2019), respectively. Gynecological and breast cancers were evaluated separately for better clarity, as breast cancer cases are the vast majority of cancer diagnoses at our department.

\section{Statistical analyses}

Descriptive statistics, such as median, mean, frequencies and percentages, were used to describe the data. Clinical characteristics were analyzed using $\chi^{2}$ and Mann-Whitney $U$ tests. For all statistical analyses, SPSS software version 26.0 was used. Two-sided $p$ values below 0.05 were regarded as significant.

\section{Ethical approval}

The ethics committee of the Medical University of Innsbruck, Austria approved the present study (IRB-Number 1163/2020). 


\section{Results}

In this study, 889 patients (495 and 394 in 2019 and 2020, respectively) with either newly diagnosed breast $(n=596)$ or gynecological $(n=293)$ cancer were included.

During the lockdowns, screening examinations and hospital bed capacity were reduced. This led to a strong decline of newly diagnosed cancers during the first and second lockdown: $-45 \%$ in gynecological cancer and $-52 \%$ in newly diagnosed breast cancer compared to the same period in 2019.

Patients' characteristics, tumor type and stage as well as clinical parameters are presented for breast cancer in Table 1 and for gynecological tumors in Table 2.

Analysis of 2019 and 2020 revealed a decrease of 70 and $35 \%$, respectively, of newly diagnosed gynecological and breast cancers in particular during the first lockdown (Fig. 1). According to the above, the number of newly diagnosed gynecological cancer cases was remarkably lower in 2020 compared to 2019 during the first lockdown period $(-45 \%)$ as well as between the two lockdowns $(-44 \%)$. An inverse correlation was found between the number of positively tested COVID-19 patients in the federal state of Tyrol (Austria) and newly diagnosed breast cancer cases at our department during 2020.

\section{Sub-analysis of breast cancer patients}

Compared to the analogue period of 2019, breast cancer patients reported significantly more tumor-associated symptoms $(55 \%$ vs. $31 \%, p=0.013)$ during and in between ( $48 \%$ vs. $32 \%, p=0.022$ ) the lockdowns (Table 1 ). In contrast to 2019 , patients were less likely to be referred by a specialist, whereas an increase of self-referring patients, due to symptoms, was noted. Furthermore, patients' tumor stage during the lockdown varied significantly compared to 2019 ( $p=0.047)$. Despite being insignificant, fewer patients underwent immediate surgery $(-11 \%)$, whereas an increase of neoadjuvant chemotherapy $(+10 \%)$ was indicated in this sub-cohort (Table 1). The drop of newly diagnosed cancer cases during first lockdown was followed by an increased number of diagnosis in between the lockdowns $(+6 \%)$.

\section{Sub-analysis of gynecological cancer patients}

As seen in the breast cancer subgroup, an increase of patients presenting themselves with tumor-associated symptoms $(+20 \%, p=0.384)$ was noted during the lockdown, despite being insignificant (Table 2). Between the two lockdown periods, a significant leap $(+25 \%)$ of selfreferring symptomatic patients was observed $(p<0.001)$ (Table 2).

Overall, the tumor stage did not differ significantly between the two analyzed years (Table 2). However, a drop in newly diagnosed cervical cancer (18\% vs. $6 \%)$ was observed. During the lockdown periods, a trend towards neoadjuvant chemotherapy ( $+8 \%$; $p=0.677)$, as well as a nonsignificant decrease in primary surgical treatment $(-12 \%$; $p=0.559$ ) was reported (Table 2).

A significant increase of patients without leading comorbidities was seen in the gynecological cancer cohort during the lockdowns $(p=0.010)$, whereas this change remained insignificant in the breast cancer subgroup $(p=0.102)$.

\section{COVID-19 screening}

Routine preoperative COVID-19 screening was performed in 296 patients leading to the detection of 3 positive COVID-19 asymptomatic patients (1\%). Of those, one was diagnosed with breast cancer and two with gynecological cancer (endometrial and ovarian cancer). For two of them, surgery had to be postponed for 3 weeks until they reached a negative test result. The third patient (ovarian cancer) was admitted to our department and undergone paracentesis but denied further treatment irrespective of her COVID-19 infection.

\section{Discussion}

In the current study, we found a strong decline of newly diagnosed gynecological and breast cancer cases in 2020 as compared to 2019. This decrease was mainly due to a lower cancer detection rate at our department during the two lockdown periods (16 March 2020-30 April 2020 and 3 November 2020-31 December 2020) as compared to the same period in 2019.

To guarantee adequate patient care, considerable efforts have been made during the first lockdown to facilitate gynecological screening examinations with a minimized risk of a hospital acquired infection: COVID-19 symptom screening by questionnaires, temperature measurements, mandatory facemasks and optimized patients waiting time. By 14th of June, these actions were expanded by COVID-19 screening of every admitted patient. Therefore, although rising infection rates were experienced during fall and winter, screening examinations and outpatient care were guaranteed. However, as our study shows, even with optimized pandemic management, a strong decline in newly diagnosed cancers was still observed throughout the year 2020 as compared to 2019 .

In the group of breast cancer, a strong decline of cancer detection rate was observed during both lockdown periods. 
Table 1 Breast cancer patients' characteristics

\begin{tabular}{|c|c|c|c|c|c|c|c|c|}
\hline Parameter & $\begin{array}{l}16 \text { Mar }-30 \\
\text { Apr and } 3 \\
\text { Nov-31 Dec } \\
2019\end{array}$ & $\begin{array}{l}16 \text { Mar }-30 \\
\text { Apr and } 3 \\
\text { Nov-31 Dec } \\
2020\end{array}$ & Difference $(\%)$ & $p$ value & $\begin{array}{l}1 \text { May-2 Nov } \\
2019\end{array}$ & $\begin{array}{l}1 \text { May-2 Nov } \\
2020\end{array}$ & Difference (\%) & $p$ value \\
\hline \multicolumn{9}{|l|}{$\begin{array}{l}\text { Breast cancer } \\
\text { patients diag- } \\
\text { nosed }\end{array}$} \\
\hline $\begin{array}{l}\text { Number of } \\
\text { patients }\end{array}$ & 115 & 55 & $-52 \%$ & & 148 & 157 & $+6 \%$ & \\
\hline $\begin{array}{l}\text { Age, median } \\
\text { (range) }\end{array}$ & $63(32-90)$ & $60(28-93)$ & $-5 \%$ & $p=0.145^{*}$ & $59(21-90)$ & $62(25-96)$ & $+5 \%$ & $p=0.035^{*}$ \\
\hline \multicolumn{9}{|l|}{$\begin{array}{l}\text { Reported } \\
\text { tumor- } \\
\text { associated } \\
\text { symptoms at } \\
\text { diagnosis }\end{array}$} \\
\hline No & $78(68 \%)$ & $25(45 \%)$ & $-23 \%$ & $p=\mathbf{0 . 0 1 3}^{\dagger}$ & $98(67 \%)$ & $81(51 \%)$ & $-16 \%$ & $p=0.022^{\dagger}$ \\
\hline Yes & $36(31 \%)$ & $30(55 \%)$ & $+24 \%$ & & $48(32 \%)$ & $75(48 \%)$ & $+16 \%$ & \\
\hline Unknown & $1(1 \%)$ & $0(0 \%)$ & & & $2(1 \%)$ & $1(1 \%)$ & & \\
\hline \multicolumn{9}{|l|}{$\begin{array}{l}\text { Referral of } \\
\text { patients }\end{array}$} \\
\hline $\begin{array}{l}\text { Patients } \\
\text { themselves } \\
\text { without } \\
\text { symptoms }\end{array}$ & $4(3 \%)$ & $1(2 \%)$ & $-1 \%$ & $p=0.288^{\dagger}$ & $7(5 \%)$ & $8(5 \%)$ & $0 \%$ & $p=0.371^{\dagger}$ \\
\hline $\begin{array}{l}\text { Patients them- } \\
\text { selves with } \\
\text { symptoms }\end{array}$ & $13(11 \%)$ & $12(21 \%)$ & $+10 \%$ & & $11(8 \%)$ & $16(10 \%)$ & $+2 \%$ & \\
\hline Specialist & $94(82 \%)$ & $40(73 \%)$ & $-9 \%$ & & $123(83 \%)$ & $131(84 \%)$ & $+1 \%$ & \\
\hline $\begin{array}{l}\text { General practi- } \\
\text { tioner }\end{array}$ & $3(3 \%)$ & $1(2 \%)$ & $-1 \%$ & & $5(3 \%)$ & $2(1 \%)$ & $-2 \%$ & \\
\hline Emergency & $0(0 \%)$ & $1(2 \%)$ & $+2 \%$ & & $0(0 \%)$ & $0(0 \%)$ & $0 \%$ & \\
\hline Unknown & $1(1 \%)$ & $0(0 \%)$ & & & $2(1 \%)$ & $0(0 \%)$ & & \\
\hline \multicolumn{9}{|l|}{$\begin{array}{l}\text { Leading } \\
\text { comorbidities }\end{array}$} \\
\hline None & $55(47 \%)$ & $35(64 \%)$ & $+17 \%$ & $p=0.077^{\dagger}$ & $84(57 \%)$ & $83(53 \%)$ & $-4 \%$ & $p=0.117^{\dagger}$ \\
\hline $\begin{array}{l}\text { Cardiovascu- } \\
\text { lar disease }\end{array}$ & $28(24 \%)$ & $10(18 \%)$ & $-6 \%$ & & $22(15 \%)$ & $38(24 \%)$ & $+9 \%$ & \\
\hline $\begin{array}{c}\text { Malignant } \\
\text { disease }\end{array}$ & $6(5 \%)$ & $5(9 \%)$ & $+4 \%$ & & $13(9 \%)$ & $14(9 \%)$ & $0 \%$ & \\
\hline $\begin{array}{l}\text { Respiratory } \\
\text { disease }\end{array}$ & $4(4 \%)$ & $0(0 \%)$ & $-4 \%$ & & $6(4 \%)$ & $8(5 \%)$ & $+1 \%$ & \\
\hline $\begin{array}{l}\text { Infectious } \\
\text { disease }\end{array}$ & $0(0 \%)$ & $0(0 \%)$ & $0 \%$ & & $4(3 \%)$ & $2(1 \%)$ & $-2 \%$ & \\
\hline $\begin{array}{l}\text { Rheumatic } \\
\text { disease }\end{array}$ & $2(2 \%)$ & $1(2 \%)$ & $0 \%$ & & $0(0 \%)$ & $0(0 \%)$ & $0 \%$ & \\
\hline $\begin{array}{l}\text { Endocrine } \\
\text { and meta- } \\
\text { bolic }\end{array}$ & $16(14 \%)$ & $1(2 \%)$ & $-12 \%$ & & $15(10 \%)$ & $6(4 \%)$ & $-6 \%$ & \\
\hline $\begin{array}{r}\text { Psychiatric } \\
\text { disorders }\end{array}$ & $2(2 \%)$ & $3(5 \%)$ & $+3 \%$ & & $3(2 \%)$ & $3(2 \%)$ & $0 \%$ & \\
\hline Others & $1(1 \%)$ & $0(0 \%)$ & $-1 \%$ & & $1(1 \%)$ & $0(0 \%)$ & $-1 \%$ & \\
\hline Unknown & $1(1 \%)$ & $0(0 \%)$ & & & $0(0 \%)$ & $3(2 \%)$ & & \\
\hline \multicolumn{9}{|l|}{$\begin{array}{r}\text { Frontline } \\
\text { therapy }\end{array}$} \\
\hline Surgery & $82(71 \%)$ & $33(60 \%)$ & $-11 \%$ & $p=0.456^{\dagger}$ & $97(66 \%)$ & $98(62 \%)$ & $-4 \%$ & $p=0.736^{\dagger}$ \\
\hline
\end{tabular}


Table 1 (continued)

\begin{tabular}{|c|c|c|c|c|c|c|c|c|}
\hline Parameter & $\begin{array}{l}16 \text { Mar }-30 \\
\text { Apr and } 3 \\
\text { Nov-31 Dec } \\
2019\end{array}$ & $\begin{array}{l}16 \text { Mar }-30 \\
\text { Apr and } 3 \\
\text { Nov-31 Dec } \\
2020\end{array}$ & Difference $(\%)$ & $p$ value & $\begin{array}{l}1 \text { May-2 Nov } \\
2019\end{array}$ & $\begin{array}{l}1 \mathrm{May}-2 \text { Nov } \\
2020\end{array}$ & Difference (\%) & $p$ value \\
\hline $\begin{array}{l}\text { Neoadjuvant } \\
\text { chemo- } \\
\text { therapy }\end{array}$ & $20(17 \%)$ & $15(27 \%)$ & $+10 \%$ & & $30(20 \%)$ & $35(22 \%)$ & $+2 \%$ & \\
\hline $\begin{array}{l}\text { Neoadjuvant } \\
\text { endocrine } \\
\text { therapy }\end{array}$ & $10(9 \%)$ & $5(9 \%)$ & $0 \%$ & & $15(10 \%)$ & $19(12 \%)$ & $+2 \%$ & \\
\hline Radiotherapy & $0(0 \%)$ & $0(0 \%)$ & $0 \%$ & & $0(0 \%)$ & $0(0 \%)$ & $0 \%$ & \\
\hline $\begin{array}{c}\text { Palliative } \\
\text { therapy }\end{array}$ & $3(3 \%)$ & $2(4 \%)$ & $+1 \%$ & & $6(4 \%)$ & $4(3 \%)$ & $-1 \%$ & \\
\hline \multicolumn{9}{|l|}{ Tumor stage } \\
\hline Tis & $10(9 \%)$ & $2(4 \%)$ & $-5 \%$ & $p=0.047^{\dagger}$ & $14(9 \%)$ & $11(7 \%)$ & $-2 \%$ & $p=0.708^{\dagger}$ \\
\hline $\mathrm{T} 1$ & $72(62 \%)$ & $24(43 \%)$ & $-19 \%$ & & $78(53 \%)$ & $81(52 \%)$ & $-1 \%$ & \\
\hline $\mathrm{T} 2$ & $17(15 \%)$ & $13(24 \%)$ & $+9 \%$ & & $34(23 \%)$ & $33(21 \%)$ & $-2 \%$ & \\
\hline $\mathrm{T} 3$ & $7(6 \%)$ & $6(11 \%)$ & $+5 \%$ & & $13(9 \%)$ & $23(15 \%)$ & $+6 \%$ & \\
\hline $\mathrm{T} 4$ & $1(1 \%)$ & $3(6 \%)$ & $+5 \%$ & & $7(5 \%)$ & $7(4 \%)$ & $-1 \%$ & \\
\hline Unknown & $8(7 \%)$ & $7(12 \%)$ & & & $2(1 \%)$ & $2(1 \%)$ & & \\
\hline
\end{tabular}

16 March 2020-30 April 2020 and 3 November 2020-31 December 2020 refers to the lockdown periods 1 and 2, respectively. 1 May 2020-2 November 2020 refers to the time between the two lockdowns

*Mann-Whitney $U$ test

${ }^{\dagger} \chi 2$ test

In between the lockdowns, cases of newly diagnosed breast cancer increased and even reached a higher number as compared to 2019. We suppose that patients who missed screening examinations during the first lockdown, tended to catch up their appointment as soon as the pandemic situation looked stabilized. Significantly more patients with tumor-specific symptoms presented directly at our department or were referred by their gynecologist due to these symptoms. This observation indicates that tumor-specific symptoms forced patients to consult a doctor, while nonsymptomatic cancer cases in patients who missed the routine mammography remained undetected. The observed tumor stage shift towards higher stage at diagnosis might also be explained by postponed mammography during the first lockdown. However, further studies on larger population are needed to prove this assumption. Moreover, there was an increasing trend towards neoadjuvant chemotherapy and a reduction in primary surgical cases during the lockdown periods. This might be explained by advanced tumor stage and the attempt to increase the overall hospitals' bed capacity.

A decrease of $45 \%$ of newly diagnosed gynecological cancers was observed as compared to 2019. In particular, nonsymptomatic tumors such as cervical cancer was underdetected in 2020, as annual PAP test was postponed during the first lockdown period. In the same time, tumor patients with tumor-specific symptoms such as postmenopausal bleeding or abdominal pain, less frequently consulted their specialist and presented themselves directly at our department.

Looking at percentages we have a similar loss in newly diagnosed tumors during the lockdown period in the breast cancer $(-52 \%)$ as in the gynecological cancer group (-45\%). In the breast cancer group, we have seen a strong decline in tumor diagnoses during the both lockdown periods and an increase in between the lockdowns. Whereas in gynecological cancer, we can just see a decline in percentage of cancer diagnoses throughout the year. The reason could be, that in gynecological cancer, we summarized different tumor entities and have a smaller sample size.

In accordance with our results, analyses of the national cancer screening patterns in the USA noticed a drop in breast and cervical cancer screening of 94\% [4]. Among calculations, the COVID-19 pandemic could lead to 36000 delayed or missed breast cancer diagnoses and 2500 missed cases of cervical cancer in the three months from March to June 2020 [5]. Moreover, strong decline in newly diagnosed cancers was observed in breast, colorectal, lung and prostate cancer in the USA and across Europe [6-9]. Kaufman et al. have shown a decrease in mean weekly numbers of newly diagnosed patients with colorectal cancer, lung cancer, pancreatic cancer, gastric cancer, esophageal cancer and breast cancer of $46.4 \%$ for the timespan of 1 March to 18 April of 2020 when compared to the weekly numbers between 6 January 2019 and 29 February 2020. For breast cancer alone, 
Table 2 Gynecological cancer patients' characteristics

\begin{tabular}{|c|c|c|c|c|c|c|c|c|}
\hline Parameter & $\begin{array}{l}16 \text { Mar-30 } \\
\text { Apr } 30 \text { and } 3 \\
\text { Nov-31 Dec } \\
2019\end{array}$ & $\begin{array}{l}16 \text { Mar-30 } \\
\text { Apr } 30 \text { and } 3 \\
\text { Nov-31 Dec } \\
2020\end{array}$ & Difference $(\%)$ & $p$ value & $\begin{array}{l}1 \text { May-2 Nov } \\
2019\end{array}$ & $\begin{array}{l}1 \text { May-2 Nov } \\
2020\end{array}$ & Difference $(\%)$ & $p$ value \\
\hline \multicolumn{9}{|l|}{$\begin{array}{l}\text { Gynecologi- } \\
\text { cal cancer } \\
\text { patients } \\
\text { diagnosed }\end{array}$} \\
\hline $\begin{array}{c}\text { Number of } \\
\text { patients }\end{array}$ & 29 & 16 & $-45 \%$ & & 102 & 57 & $-44 \%$ & \\
\hline $\begin{array}{l}\text { Age, median } \\
\text { (range) }\end{array}$ & $60(34-83)$ & $66(28-93)$ & $+10 \%$ & $p=0.164^{*}$ & $61(25-96)$ & $62(30-95)$ & $+1 \%$ & $p=0.568^{*}$ \\
\hline \multicolumn{9}{|c|}{ Reported tumor-associated symptoms at diagnosis } \\
\hline No & $5(17 \%)$ & $1(6 \%)$ & $-11 \%$ & $p=0.426^{\dagger}$ & $23(22 \%)$ & $19(33 \%)$ & $+11 \%$ & $p=0.170^{\dagger}$ \\
\hline Yes & $16(55 \%)$ & $12(75 \%)$ & $+20 \%$ & & $60(59 \%)$ & $34(60 \%)$ & $+1 \%$ & \\
\hline Unknown & $8(28 \%)$ & $3(19 \%)$ & & & $19(19 \%)$ & $4(7 \%)$ & & \\
\hline \multicolumn{9}{|c|}{ Referral of patients } \\
\hline $\begin{array}{l}\text { Patients } \\
\text { themselves } \\
\text { without } \\
\text { symptoms }\end{array}$ & $1(3 \%)$ & $0(0 \%)$ & $-3 \%$ & $p=0.118^{\dagger}$ & $0(0 \%)$ & $0(0 \%)$ & $0 \%$ & $p<0.001^{\dagger}$ \\
\hline $\begin{array}{l}\text { Patients them- } \\
\text { selves with } \\
\text { symptoms }\end{array}$ & $0(0 \%)$ & $0(0 \%)$ & $0 \%$ & & $3(3 \%)$ & $16(28 \%)$ & $+25 \%$ & \\
\hline Specialist & $20(69 \%)$ & $13(81 \%)$ & $+12 \%$ & & $79(78 \%)$ & $40(70 \%)$ & $-8 \%$ & \\
\hline $\begin{array}{l}\text { General prac- } \\
\text { titioner }\end{array}$ & $2(8 \%)$ & $3(19 \%)$ & $+11 \%$ & & $6(6 \%)$ & $1(2 \%)$ & $-4 \%$ & \\
\hline Emergency & $3(10 \%)$ & $0(0 \%)$ & $-10 \%$ & & $2(2 \%)$ & $0(0 \%)$ & $-2 \%$ & \\
\hline Unknown & $3(10 \%)$ & $0(0 \%)$ & & & $12(11 \%)$ & $0(0 \%)$ & & \\
\hline \multicolumn{9}{|l|}{ Comorbidities } \\
\hline None & $6(20 \%)$ & $3(19 \%)$ & $-1 \%$ & $p=0.751^{\dagger}$ & $28(28 \%)$ & $26(46 \%)$ & $-18 \%$ & $p=\mathbf{0 . 0 1 0} \dagger$ \\
\hline Yes & $16(55 \%)$ & $11(69 \%)$ & $-14 \%$ & & $53(52 \%)$ & $29(51 \%)$ & $-1 \%$ & \\
\hline Unknown & $7(25 \%)$ & $2(12 \%)$ & & & $21(20 \%)$ & $2(3 \%)$ & & \\
\hline \multicolumn{9}{|c|}{ Frontline therapy } \\
\hline Operation & $22(75 \%)$ & $10(63 \%)$ & $-12 \%$ & $p=0.559^{\dagger}$ & $68(66 \%)$ & $43(74 \%)$ & $+8 \%$ & $p=0.677^{\dagger}$ \\
\hline $\begin{array}{l}\text { Neoadjuvant } \\
\text { chemo- } \\
\text { therapy }\end{array}$ & $3(11 \%)$ & $3(19 \%)$ & $+8 \%$ & & $17(17 \%)$ & $8(14 \%)$ & $-3 \%$ & \\
\hline $\begin{array}{l}\text { Neoadjuvant } \\
\text { endocrine } \\
\text { therapy }\end{array}$ & $0(0 \%)$ & $0(0 \%)$ & $0 \%$ & & $0(0 \%)$ & $0(0 \%)$ & $0 \%$ & \\
\hline Radiation & $0(0 \%)$ & $0(0 \%)$ & $0 \%$ & & $2(2 \%)$ & $1(2 \%)$ & $0 \%$ & \\
\hline $\begin{array}{l}\text { Combined } \\
\text { chemoradia- } \\
\text { tion }\end{array}$ & $3(11 \%)$ & $1(6 \%)$ & $-5 \%$ & & $11(11 \%)$ & $2(4 \%)$ & $-7 \%$ & \\
\hline $\begin{array}{l}\text { COVID } \\
\text { associated } \\
\text { treatment } \\
\text { delay }\end{array}$ & $0(0 \%)$ & $0(0 \%)$ & $0 \%$ & & $0(0 \%)$ & $0(0 \%)$ & $0 \%$ & \\
\hline $\begin{array}{l}\text { Palliative } \\
\text { therapy }\end{array}$ & $0(0 \%)$ & $1(6 \%)$ & $+6 \%$ & & $3(3 \%)$ & $2(4 \%)$ & $+1 \%$ & \\
\hline Unknown & $1(3 \%)$ & $1(6 \%)$ & & & $1(1 \%)$ & $1(2 \%)$ & & \\
\hline
\end{tabular}


Table 2 (continued)

\begin{tabular}{|c|c|c|c|c|c|c|c|c|}
\hline Parameter & $\begin{array}{l}16 \text { Mar-30 } \\
\text { Apr } 30 \text { and } 3 \\
\text { Nov-31 Dec } \\
2019\end{array}$ & $\begin{array}{l}16 \text { Mar-30 } \\
\text { Apr } 30 \text { and } 3 \\
\text { Nov-31 Dec } \\
2020\end{array}$ & Difference $(\%)$ & $p$ value & $\begin{array}{l}1 \text { May-2 Nov } \\
2019\end{array}$ & $\begin{array}{l}1 \text { May-2 Nov } \\
2020\end{array}$ & Difference $(\%)$ & $p$ value \\
\hline \multicolumn{9}{|l|}{ Diagnosis } \\
\hline $\begin{array}{r}\text { Ovarian } \\
\text { cancer }\end{array}$ & $13(45 \%)$ & $9(56 \%)$ & $+11 \%$ & $p=0.542^{\dagger}$ & $40(39 \%)$ & $23(40 \%)$ & $+1 \%$ & $p=0.558^{\dagger}$ \\
\hline $\begin{array}{l}\text { Endometrial } \\
\text { cancer }\end{array}$ & $7(24 \%)$ & $6(38 \%)$ & $+14 \%$ & & $29(28 \%)$ & $19(33 \%)$ & $+5 \%$ & \\
\hline $\begin{array}{c}\text { Cervical } \\
\text { cancer }\end{array}$ & $5(18 \%)$ & $1(6 \%)$ & $-12 \%$ & & $19(19 \%)$ & $5(9 \%)$ & $-10 \%$ & \\
\hline Vulva cancer & $2(7 \%)$ & $0(0 \%)$ & $-7 \%$ & & $6(6 \%)$ & $6(10 \%)$ & $+4 \%$ & \\
\hline Sarcoma & $1(3 \%)$ & $0(0 \%)$ & $-3 \%$ & & $4(4 \%)$ & $2(4 \%)$ & $0 \%$ & \\
\hline Others & $1(3 \%)$ & $0(0 \%)$ & $-3 \%$ & & $4(4 \%)$ & $2(4 \%)$ & $0 \%$ & \\
\hline \multicolumn{9}{|c|}{ FIGO stage ovarian cancer } \\
\hline I & $3(23 \%)$ & $1(11 \%)$ & $-12 \%$ & $p=0.145^{\dagger}$ & $12(30 \%)$ & $6(26 \%)$ & $-4 \%$ & $p=0.795^{\dagger}$ \\
\hline II & $1(7 \%)$ & $0(0 \%)$ & $-7 \%$ & & $1(3 \%)$ & $1(4 \%)$ & $+1 \%$ & \\
\hline III & $7(55 \%)$ & $5(56 \%)$ & $+1 \%$ & & $19(48 \%)$ & $10(44 \%)$ & $-4 \%$ & \\
\hline IV & $2(15 \%)$ & $0(0 \%)$ & $-15 \%$ & & $6(15 \%)$ & $6(26 \%)$ & $+11 \%$ & \\
\hline Unknown & $0(0 \%)$ & $3(33 \%)$ & & & $2(4 \%)$ & $0(0 \%)$ & & \\
\hline \multicolumn{9}{|c|}{ FIGO stage endometrial cancer } \\
\hline I & $7(100 \%)$ & $5(83 \%)$ & $-17 \%$ & $p=0.261^{\dagger}$ & $23(80 \%)$ & $16(85 \%)$ & $+5 \%$ & $p=0.218^{\dagger}$ \\
\hline II & $0(0 \%)$ & $0(0 \%)$ & $0 \%$ & & $1(3 \%)$ & $2(10 \%)$ & $+7 \%$ & \\
\hline III & $0(0 \%)$ & $0(0 \%)$ & $0 \%$ & & $0(0 \%)$ & $1(5 \%)$ & $+5 \%$ & \\
\hline IV & $0(0 \%)$ & $0(0 \%)$ & $0 \%$ & & $3(10 \%)$ & $0(0 \%)$ & $-10 \%$ & \\
\hline Unknown & $0(0 \%)$ & $1(17 \%)$ & & & $2(7 \%)$ & $0(0 \%)$ & & \\
\hline
\end{tabular}

16 March 2020-30 April 2020 and 3 November 2020-31 December 2020 refers to the lockdown periods 1 and 2, respectively. 1 May 2020-2 November 2020 refers to the time between the two lockdowns

*Mann-Whitney $U$ test

${ }^{\dagger} \chi^{2}$ test

Fig. 1 Impact of COVID-19 lockdown on newly diagnosed cancers. Numbers of newly diagnosed breast and gynecological cancers per month in 2020 compared to the same periods in 2019. Pre-lockdown refers to 1 January-15 March, lockdown 1 to 16 March- 30 April, between lockdowns to 1 May-2 November and lockdown 2 to 3 November-31 December

\section{Newly diagnosed gynecological and breast cancer in relation to COVID-19 cases}

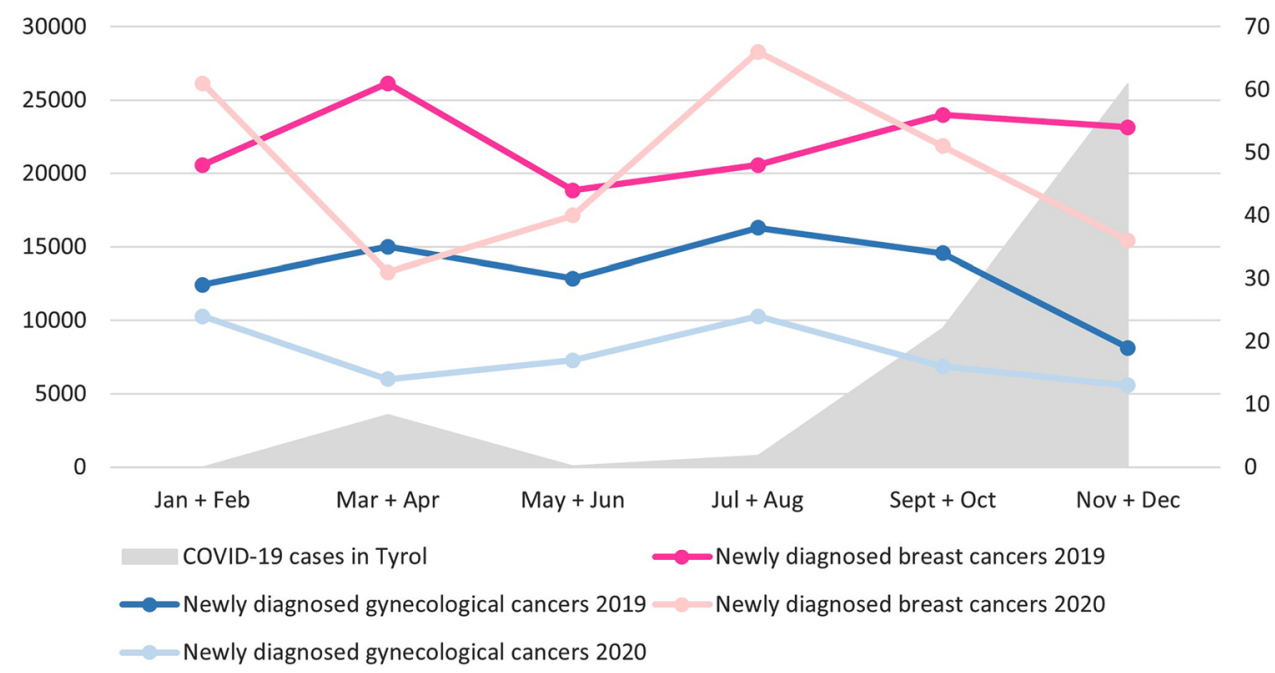


they describe a decrease of $51.8 \%$ in the same period [10]. Also, Morais et al. were able to show a decrease of nearly $40 \%$ in new cases of 12 different types of cancer including breast cancer and cervical cancer. In their study, cervical cancer has shown the highest decrease with $74.3 \%$ when compared to the year 2019. For breast cancer, a decrease of $38.6 \%$ was found [11].

In contrast to most other published literature, this study includes data of the whole year 2020, compared to 2019. By investigating the whole year, we were able to find an inverse correlation of the number of positively tested COVID-19 patients in Austria and newly diagnosed cancers at our department. Thus, we analysed not only the differences in cancer diagnoses during the lockdown, but also investigated potential short term increases in between the lockdowns, as shown in our sub-analysis for breast cancer patients.

Efforts have been taken to organize cancer screening and management during the pandemic including the implementation of telemedicine for the outpatient treatment of cancer survivors to minimize face-to-face appointments [12]. It is recommended to continue oncological surgery, chemotherapy and radiotherapy based on priorities, while surgeries due to benign diseases should be postponed [13].

Treatment delays in potentially curable disease could lead to inferior outcomes and have impact on the overall survival of our patients, with the risk of missing the optimal treatment window. As the COVID-19 pandemic will be a challenge for some time to come, new strategies in patient care are needed to minimize the risk of infection. New strategies may include telemedicine or self-sampling HPV test, which detects viral nucleic acid rather than morphological changes and thus does not rely on healthcare practitioners visualizing the cervix [14], and thereby provide early diagnosis and improved treatment options for our patients. Most importantly, awareness must be raised for the importance of screening examinations to avoid any further shift in tumor stages at the time of diagnosis.

The major limitations of the current study are its relatively small sample size of only 889 patients and its singlecenter observational character. Despite these limitations, we were able to demonstrate that the COVID-19 pandemic led to a strong decline in the detection rate of newly diagnosed gynecological and breast cancers, which is in accordance with the findings of other subspecialities.

Author contributions IT and CM: protocol/project development. KK, ER, KL, CE, JK: data collection and management. KK, ER, IT: data analysis. KK, ER, KL, CE, JK, IT, CM: manuscript writing/editing.

Funding Open access funding provided by University of Innsbruck and Medical University of Innsbruck.
Availability of data and materials Data available on request due to privacy/ethical restrictions.

Code availability Not applicable.

\section{Declarations}

Conflict of interest The authors declare no conflict of interest.

Ethics approval The ethics committee of the Medical University of Innsbruck, Austria approved the present study (IRB-Number $1163 / 2020)$

Consent to participate Not applicable.

Consent for publication Not applicable.

Open Access This article is licensed under a Creative Commons Attribution 4.0 International License, which permits use, sharing, adaptation, distribution and reproduction in any medium or format, as long as you give appropriate credit to the original author(s) and the source, provide a link to the Creative Commons licence, and indicate if changes were made. The images or other third party material in this article are included in the article's Creative Commons licence, unless indicated otherwise in a credit line to the material. If material is not included in the article's Creative Commons licence and your intended use is not permitted by statutory regulation or exceeds the permitted use, you will need to obtain permission directly from the copyright holder. To view a copy of this licence, visit http://creativecommons.org/licenses/by/4.0/.

\section{References}

1. John Hopkins University and Medicine (2020) COVID-19 MapJohns Hopkins Coronavirus Resource Center. 2020 [cited 26 Jan 2021]. https://coronavirus.jhu.edu/map.html.

2. Federal Ministry of Social Affairs, Healthcare and Consumer Protection, Republic of Austria (2020) Official COVID19 Dashboard (Public Information): hospitalisation [cited 26 Jan 2021]. https:// info.gesundheitsministerium.at/dashboard_Hosp.html?l=de.

3. Tsibulak I, Reiser E, Bogner G, Petru E, Hell-Teutsch J, Reinthaller A et al (2020) Decrease in gynecological cancer diagnoses during the COVID-19 pandemic: an Austrian perspective. Int J Gynecol Cancer 30(11):1667-1671

4. EPIC Health Research Network (EHRN) Preventive cancer screenings during COVID-19 pandemic (2020). Accessed 12 June 2020. https://ehrn.org/wp-content/uploads/Preventive-Cancer-Screenngs-during-COVID-19-Pandemic.pdf

5. Gorin SNS, Jimbo M, Heizelman R, Harmes KM, Harper DM (2021) The future of cancer screening after COVID-19 may be at home. Cancer 127(4):498-503. https://doi.org/10.1002/cncr. 33274 (Epub 10 Nov 2020; PMID: 33170520)

6. Patt D, Gordan L, Diaz M, Okon T, Grady L, Harmison M et al (2020) Impact of COVID-19 on cancer care: how the pandemic is delaying cancer diagnosis and treatment for American seniors. JCO Clin Cancer Inform 4:1059-1071

7. Turnbull C (2021) Effect of COVID-19 on colorectal cancer care in England. Lancet Gastroenterol Hepatol S2468-1253(21):00017. https://doi.org/10.1016/S2468-1253(21)00017-0

8. Bakouny Z, Paciotti M, Schmidt AL, Lipsitz SR, Choueiri TK, Trinh QD (2021) Cancer screening tests and cancer diagnoses 
during the COVID-19 pandemic. JAMA Oncol. https://doi.org/ 10.1001/jamaoncol.2020.7600

9. Oldani C, Vanni G, Buonomo OC (2020) COVID-19 unintended effects on breast cancer in Italy after the great lockdown. Front Public Health 8:601748. https://doi.org/10.3389/fpubh.2020. 601748

10. Kaufman HW, Chen Z, Niles J, Fesko Y (2020) Changes in the number of US patients with newly identified cancer before and during the coronavirus disease 2019 (COVID-19) pandemic. JAMA Netw Open 3(8):e2017267. https://doi.org/10.1001/jaman etworkopen.2020.17267.)

11. Morais S, Antunes L, Rodrigues J, Fontes F, Bento MJ, Lunet N (2021) The impact of the coronavirus disease 2019 pandemic on the diagnosis and treatment of cancer in Northern Portugal. Eur $\mathbf{J}$ Cancer Prev. https://doi.org/10.1097/CEJ.0000000000000686

12. Mancebo G, Solé-Sedeño JM, Membrive I, Taus A, Castells M, Serrano L et al (2020) Gynecologic cancer surveillance in the era of SARS-CoV-2 (COVID-19). Int J Gynecol Cancer. https://doi. org/10.1136/ijgc-2020-001942

13. Uwins C, Bhandoria GP, Shylasree TS, Butler-Manuel S, Ellis P, Chatterjee J et al (2020) COVID-19 and gynecological cancer: a review of the published guidelines. Int J Gynecol Cancer. https:// doi.org/10.1136/ijgc-2020-001634

14. Hawkes D, Keung MHT, Huang Y, McDermott TL, Romano J, Saville M, Brotherton JML (2020) Self-collection for cervical screening programs: from research to reality. Cancers 12(4):1053. https://doi.org/10.3390/cancers12041053

Publisher's Note Springer Nature remains neutral with regard to jurisdictional claims in published maps and institutional affiliations. 